\title{
Causes of consultations and results of radiological and ultrasound methods in lizard diseases (2006-2014)
}

\author{
ANNA ŁOJSZCZYK-SZCZEPANIAK, KLAUDIUSZ O. SZCZEPANIAK*, \\ MACIEJ GRZYBEK*, BARBARA LISIAK
}

\begin{abstract}
Laboratory of Radiology and Ultrasonography, Department and Clinic of Animal Surgery, Faculty of Veterinary Medicine, University of Life Sciences in Lublin, Głęboka 30, 20-612 Lublin, Poland *Sub-Department of Parasitology and Invasive Diseases, Institute of Biological Bases of Animal Diseases, Faculty of Veterinary Medicine, University of Life Sciences in Lublin, Akademicka 12, 20-950 Lublin, Poland
\end{abstract}

\section{Łojszczyk-Szczepaniak A., Szczepaniak K. O., Grzybek M., Lisiak B. Causes of consultations and results of radiological and ultrasound methods in lizard diseases (2006-2014)}

\section{Summary}

Reptiles are becoming increasingly popular animals in amateur husbandry in Poland and worldwide. The world literature, however, does not provide data on the actual causes of radiological and ultrasound consultations in certain groups of animals, including reptiles. The aim of the study was the analysis of causes and results of diagnostic imaging examinations. The study was based on the data of 75 lizards which were patients of the university diagnostic imaging service in the years 2006-2014.

The most common clinical signs in those animals were visible body deformations (33.33\%), loss of body weight $(30.67 \%)$ and loss of appetite $(26.67 \%)$. In cases in which a preliminary diagnosis had been made, the largest proportion of animals were referred with suspected post-traumatic changes (12\%), dystocia $(10.67 \%)$ and ileus $(9.33 \%)$. In diagnostic imaging tests, musculoskeletal $(29.33 \%)$ and coelomic abnormalities $(\mathbf{4 0 . 3 3 \% )}$ were most commonly confirmed. Coelomic changes affected mainly the liver, reproductive system and gastrointestinal tract. For the musculoskeletal system, the most frequently identified causes were the presence of post-traumatic changes, malformations of the vertebral column, as well as metabolic bone disease. Cardiorespiratory and urinary problems were observed only sporadically. In twenty-two animals (29.33\%), radiographic and ultrasound examinations failed to establish a diagnosis.

The results of this study provide valuable data on the cross-section of the most common problems leading to diagnostic imaging evaluation in lizards. This research demonstrates that, despite the growing popularity of exotic animals, most of the problems diagnosed in such animals still arise from inappropriate breeding and housing conditions. In most cases, diagnostic imaging provides valuable information, making it possible to implement further steps.

Keywords: reptiles, exotic species, radiology, ultrasound

Reptiles are becoming increasingly popular animals in amateur husbandry. Growing attention is being focused on their health problems, as well as on diagnostic capabilities $(3,19)$. However, diagnostic imaging of these animals can be problematic, as reptiles are characterized by large anatomic variability $(1,16,17)$. The lack of a diaphragm, the peculiar topography of internal organs, and the small amount of intraperitoneal adipose tissue between parenchymal organs negatively affect image clarity in radiological examinations (17). In addition, there are no objective criteria for image evaluation with respect to the normal organ size (1, $13,16,17)$.

The world literature, however, does include publications on correct tomographic, radiological and ultrasound images for particular groups of reptiles (1-3). The majority of publications are based on case studies of individual animals, selected groups of animals or suborders of snakes and lizards and orders of turtles. At the same time, the world literature does not provide data on actual causes of radiological and ultrasound consultations in certain groups of animals, based on 
a retrospective analysis of the consulted cases. Nor do the studies analyze the effectiveness of these consultations in particular cases. This suggests that what is needed is an approach that makes it possible to objectively analyze health problems affecting these animals and the possibilities of diagnostic imaging in this field.

The aim of the study was to analyze the causes and results of radiological and ultrasound consultations concerning lizards in the years 2006-2014. This research paper discusses the most common clinical indications based on clinical signs and initial diagnosis, which are the cause of referral for radiographic and ultrasonographic examinations. The results and accuracy of imaging studies were also analyzed.

\section{Material and methods}

Radiologic data were collected retrospectively between 2006 and 2014 from the archive system of the Laboratory of Radiology and Ultrasonography, Department and Clinic of Animal Surgery. All lizards referred for radiological and ultrasound examination and registered in the information system of the Laboratory of Radiology and Ultrasonography were included to the study. All animals had written referrals with fields to fill, including the owner's name and address, animal details (species, sex, age), the anatomical area to be examined, clinical history and signs, as well as the initial diagnosis. After registration, animals were examined (X-ray and/or ultrasound examination, depending on the referral). X-ray and ultrasound images were assessed by three veterinary radiologists in their independent clinical trials. All data included information from medical referrals, as well as from the subsequent radiology reports. Followup examinations were excluded from the research, and they were counted as one visit.

On the basis of the referrals, the data obtained were classified into several categories: the number of patients examined, patients' details (generic family, species, age, sex), clinical signs (the owner's primary complaint), results of the clinical examination, the initial diagnosis and the anatomical area to be examined. If the medical referral lacked any of the required information (patients' age and sex, clinical signs, preliminary diagnosis), only the available information was taken into account. If the referring veterinarian described more than one clinical symptom, all of them were taken into account. On the basis of radiological data, the type of examinations (X-ray or ultrasound) and major diagnostic findings were graded as well. In cases in which a diagnosis had not been made, the reason was explained.

In the years 2006-2010, radiographic examinations were carried out by means of a Mobilax machine using cassettes with mammographic and high-sensitivity films (Cawo Photochemisches $\mathrm{GmbH}$ ) measuring $24 \times 30 \mathrm{~cm}$ and $30 \times 40 \mathrm{~cm}$, depending on the size of the patient. In 2010-2014, all examinations were conducted with the Leonardo DR System Vet (digital direct X-ray system). The radiological examinations were performed according to standardized protocols, in right lateral and dorso-ventral views with the central X-ray beam directed at the center of the area of interest.

Ultrasonographic examinations were performed using microconvex and linear probes, depending on the size of the animal, with a frequency range between 5 and $22 \mathrm{MHz}$. The following equipment was used for the examinations: in 2006-2010, Honda 4000 (linear probes with frequency 7.5-10 MHz, microconvex probes 3.5-7 MHz); in 2010-2013, Mindray DC 7 (linear probes 10-12 MHz, microconvex probes 6.5-7.5 MHz); and in 2013-2014, EsaoteMyLab Twice (linear probes 3-22 MHz, microconvex probes 3-9 MHz).

Data analysis was performed with a standard computer program (Microsoft Excel 2010, Microsoft Corp.). All the results are presented as percentages, rounded to two decimal places. Percentage values are given with $95 \%$ confidence limits $(95 \% \mathrm{CL})$, calculated by the bespoke software „Percentage Confidence Limits vs. 14" (courtesy of Dr. F. S. Gilbert and Prof. J. M. Behnke, University of Nottingham) based on the statistical tables of Sokal and Roholf.

\section{Results and discussion}

A total of 75 lizards were analyzed. Most of the animals referred for diagnostic imaging examination belonged to the Agamidae (44 individuals), Iguanidae (11 individuals) and Chamaeleonidae (9 individuals) families (Tab. 1).

The mean age of the animals was 27.5 months, with a minimum value of 4 months and a maximum value of 108 months ( 9 years). In $14.67 \%$ of the cases (11 animals), the age was unknown. The study group comprised 29 females, 20 males and 26 animals of unspecified sex.

The animals most often referred for examination were those with musculoskeletal problems, especially with visible body deformations (33.33\%, 25 individuals) affecting the spine (8 lizards) or other parts of the skeletal system (12 lizards), as well as those with changes in soft tissues, such as swellings or tumors (5 lizards). The other most common presenting complaints are shown in Figure 1. Often more than one clinical sign occurred simultaneously.

Initial diagnoses made by the referring veterinarians are shown in Figure 2. In 48\% of all cases, the preliminary diagnosis was not given.

During diagnostic imaging, the majority of the animals were given both radiographic and ultrasound examinations (54 ultrasound tests and $57 \mathrm{X}$-rays). All

Tab. 1. Number of patients examined

\begin{tabular}{|l|c|l|c|}
\hline Generic family & $\begin{array}{c}\text { Number of } \\
\text { lizards in the } \\
\text { generic family }\end{array}$ & Represented types & $\begin{array}{c}\text { Number of } \\
\text { individuals }\end{array}$ \\
\hline Agamidae & 44 & $\begin{array}{l}\text { Pogona vitticeps } \\
\text { Acanthozaura }\end{array}$ & 33 \\
Pogona henrylawsoni \\
Physignathus cocincinus \\
Iguanidae
\end{tabular}




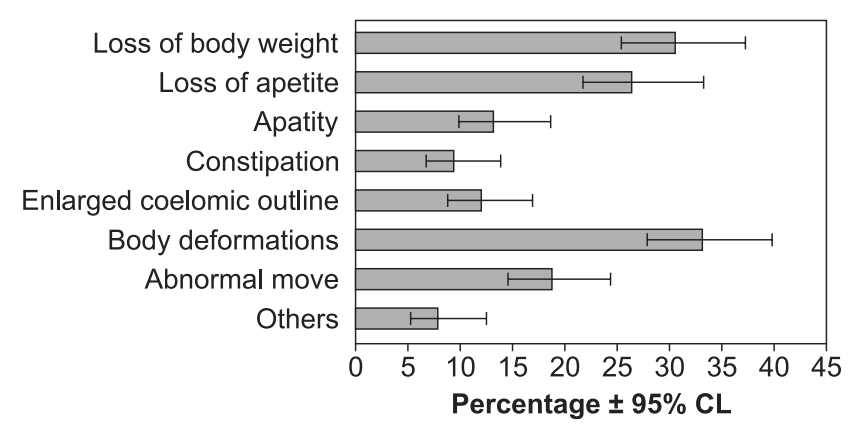

Fig. 1. The most frequent clinical signs found in animals examined

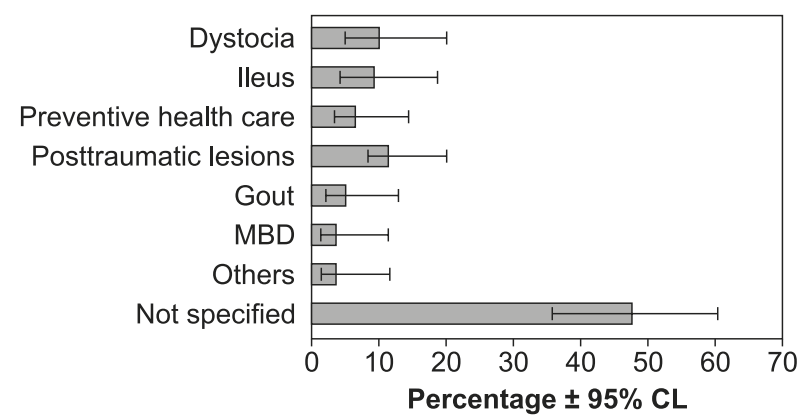

Fig. 2. Initial diagnoses made by referring veterinarians

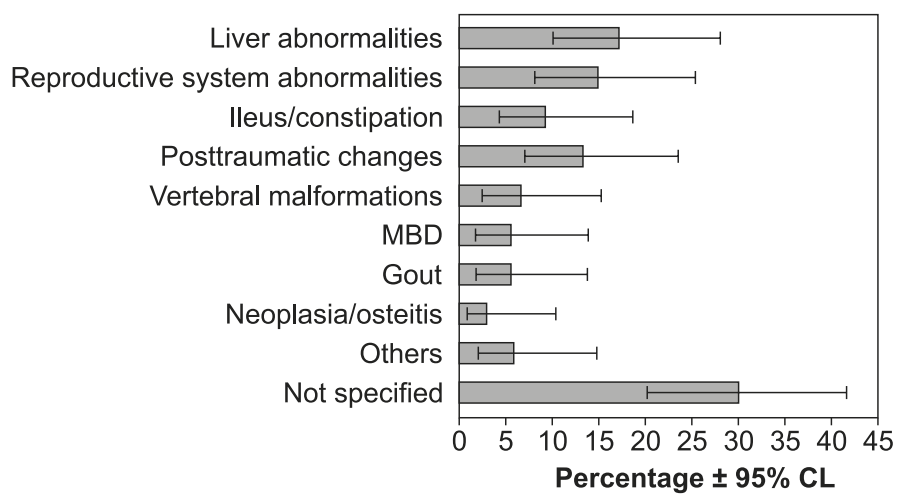

Fig. 3. Diagnostic imaging findings

diagnostic imaging findings are shown in Figure 3. Among musculoskeletal abnormalities, post-traumatic changes $(13.33 \%, 10$ individuals) were most often identified in the spine, limb bones and, in one case, jaw bones. There were also several animals with vertebral malformations $(6.67 \%, 5$ individuals), including the lordosis of the thoracic and lumbar vertebral column as well as malformations of the caudal vertebrae. In these cases, the radiopacity of the skeletal system was normal, no fractures or other bone changes being visible. These animals were juvenile, aged 9-12 months, and were kept in poor husbandry conditions. Further examination also revealed coccidiosis and adenovirosis. Other skeletal abnormalities, in this group of lizards, were diagnosed less often (Fig. 3).

Coelomic changes, which were found most often, affected mainly the liver $(17.33 \%, 13$ individuals), reproductive system $(14.67 \%, 11$ individuals $)$ and gastrointestinal tract (Tab. 2). In $13.3 \%$ of the animals (10 individuals) radiopaque elements (stones or gravel) were found in the gastrointestinal (GI) tract. Three animals were identified without radiological and ultrasound features of ileus. In seven of the animals (9.33\%) GI tract obstruction was observed.

The most frequently found conditions, diagnosed also on the basis of the animals' history and clinical signs, such as a loss of appetite, constipation and apathy, were pre-ovulatory follicle stasis and post-ovulatory follicle and egg stasis. In eight cases, X-ray and ultrasound examinations were performed, while in three cases only an ultrasound examination was done. In five cases, follicles of 1-3 cm were observed on survey radiographs, but the examination could not precisely determine their developmental stage (pre- or post- ovulatory). In most cases in which the ultrasound examination identified eggs with distinct features of shell calcification, these features were not seen on survey radiographs. In two cases, X-ray examination failed to detect eggs, and in these lizards a large amount of free coelomic fluid was observed. Echogenic free coelomic fluid was observed in one more lizard with postovulatory follicles, but this animal had undergone only an ultrasound examination. In one case, apart from eggs, decreased lung radiopacity was also found.

All cases in which free coelomic fluid was observed are shown in Table 3.

Other problems related to the coelomic cavity were found less frequently. In one 3.5-year-old Chinese water dragon, in addition to gallstones, ultrasound features of GI inflammation were observed. The lumen of the intestines was distended and fluid-filled with significantly

Tab. 2. Coelomic abnormalities in animals examined

\begin{tabular}{|l|l|l|l|}
\hline \multicolumn{1}{|c|}{$\begin{array}{c}\text { Liver } \\
\text { (13 lizards) }\end{array}$} & \multicolumn{1}{|c|}{$\begin{array}{c}\text { Reproductive system } \\
\text { (11 lizards) }\end{array}$} & \multicolumn{1}{c|}{$\begin{array}{c}\text { Gastrointestinal tract } \\
\text { (7 lizards) }\end{array}$} & \multicolumn{1}{c|}{ 0ther } \\
\hline $\begin{array}{l}\text { Ultrasound signs of biliary tract obstruction } \\
\text { - severe gallbladder distension } \\
\text { - cystic duct dilatation }\end{array}$ & Preovulatory follicle stasis & Gastrointestinal tract obstruction & Gastrointestinal tract inflammation \\
$\begin{array}{l}\text { Significant increase in liver echogenicity } \\
\text { Heterogeneous liver echogenicity }\end{array}$ & Postovulatory follicle stasis & Gravel, stones - $\mathbf{6}$ cases & Urolithiasis \\
$\begin{array}{l}\text { Liver enlargement with normal echogenicity } \\
\text { Gallstones }\end{array}$ & Nog stasis & & Gastrointestinal tract neoplasia \\
$\begin{array}{l}\text { Cholecystitis } \\
\text { - gallbladder wall oedema } \\
\text { - gallstones and debris in the gallbladder lumen } \mathbf{1} \text { case }\end{array}$ & & & \\
\hline
\end{tabular}


Tab. 3. Cases in which free fluid was found in the coelomic cavity

\begin{tabular}{|c|l|l|}
\hline Number of individuals & Type of exudate & \multicolumn{1}{|c|}{ Changes observed } \\
\hline 2 & Anechoic & Post-traumatic state \\
\hline 1 & Anechoic & Hepatomegaly \\
1 & Anechoic & Ileus \\
\hline 1 & Anechoic & Without visible changes \\
\hline 1 & Echogenic & Diffuse neoplastic disease \\
1 & Echogenic & Pneumonia \\
\hline
\end{tabular}

accelerated motility. Later laboratory tests revealed flagellate infection of the GI tract. In a 5-year-old green iguana, showing loss of appetite, dejection and aggressive behavior, urinary bladder urolithiasis was found. In a bearded dragon, advanced neoplastic disease of the gastrointestinal tract was observed. Further investigation confirmed intestinal leiomyosarcoma (11).

Cardiorespiratory problems occurred less often. In two lizards with symptoms of dyspnea, a decreased lung radiopacity was observed. One animal was examined after a fall from a height, and the other was treated for pneumonia. Besides decreased lung radiopacity, the presence of free coelomic fluid was observed as well.

In twenty-two animals (29.33\%), radiographic and ultrasound examinations did not help in establishing a diagnosis. The small size of three animals precluded reliable tests, and therefore no tests were made. In the other cases, radiography and ultrasound showed no lesions and thus did not result in a diagnosis.

Among the most common causes of radiological and ultrasound consultations in reptiles, the literature mentions diseases of the cardiovascular, skeletal, gastrointestinal and genitourinary systems $(1,7,12)$. The cases analyzed here partially confirm the literature data.

On the basis of the observations, besides the clinical signs related to the musculoskeletal system, the majority of clinical signs in exotic patients referred for diagnostic imaging consultations are general and highly non-specific. They included mainly loss of appetite, loss of body weight, apathy or constipation. These signs were usually attributed to digestive tract problems or reproductive system problems, such as ileus or dystocia, although in nearly half of the cases, preliminary diagnosis was not made.

In fact, diagnostic imaging results frequently confirmed problems related to the gastrointestinal tract, as well as the reproductive and skeletal systems. The problems identified in our research are usually mentioned in the literature as caused by poor husbandry practices, incorrect incubation temperatures, and improper nutrition $(10,19)$. For the most part, they are avoidable with proper husbandry conditions.

In the course of diagnostic imaging procedures, it was observed that ultrasound examination is much more useful than radiographic examination in the evaluation of coelomic organs in reptiles. The ultrasound provided much more information in cases of abnormalities of the bile duct, liver, and reproductive system. It also made it easy to determine the sexual status of animals, which is consistent with conclusions drawn by other authors $(1,4,6,10,17)$. These results indicate greater usefulness of ultrasound examination compared with radiographic examination, which may yield low-contrast images due to the anatomic characteristics of reptiles, especially close anatomic proximity of internal organs, a small amount of internal adipose tissue, the lack of a clearly demarcated thorax and abdomen, as well as image degradation from the superimposed exoskeleton and cutaneous scales (17). These problems are not encountered in the diagnostic imaging of mammals. On the other hand, as in cats and dogs, ultrasound rarely helps in identifying changes that are pathognomonic for a specific disease, and additional procedures, including fine needle aspiration, are recommended in these cases $(9,20)$. In the evaluation of the reproductive system, the small size of animals and low-contrast images made it impossible to differentiate the developmental stages of eggs by X-ray examination. This is also consistent with findings of other authors, who report radiological assessment as being very useful in the case where postovulatory follicles measured about $2 \mathrm{~cm}$ (5). In cases in which dystocia and follicular stasis were suspected, the results of ultrasonographic examinations were also considered together with the clinical signs and clinical examination results.

The radiological examination of the coelomic organs was very useful in the evaluation of the digestive tract. It showed the presence of radiopaque foreign bodies and features of ileus. Radiopaque elements were visible in the gastrointestinal tract in many reptiles. They were mostly fragments of terrarium substrates, which, as the literature suggests, may have been accidentally ingested with food and their role is to aid digestion (1, $17,19)$. They may cause constipation or ileus only under abnormal conditions, in the presence of additional factors, such as dehydration and physical inactivity, spine injuries, functional changes in the reproductive system, tumors and parasites (17). Therefore a detailed assessment is very important in these cases. The ultrasound examination is a complementary method to radiography in the evaluation of gastrointestinal tract. According to our findings, the exception were very small patients, in which the presence of large structures with strong acoustic shadows almost completely prevented the visualization of GI tract structures. In such cases, X-ray examination played the primary role.

Although the literature includes cardiovascular and urinary system diseases among the most common causes of radiological and ultrasound consultations in reptiles, in our clinic these problems were observed only occasionally (1). Research into the respiratory system has not yet been conducted on a large group of animals either. In radiological examinations performed for this study, reduced local lung tissue aeration in the form of hetero- or homogeneous radiopacity was observed in 
three animals. There were difficulties in the assessment of pulmonary patterns in the animals, and the diagnoses were made on the basis of clinical examination results and other diagnostic imaging examinations. According to Silverman, inflammatory conditions of the lungs manifest themselves by increased tissue shadow, which should be homogenous in lizards under normal conditions, with possible vascular markings (17). In one lizard, the increased lung radiopacity was related to post-traumatic contusio pulmonum, but these changes had a similar appearance to pneumonia lesions in another animal. Similar problems were observed by others authors, and for this reason computed tomography is regarded as the gold standard in assessing the lungs and heart in reptiles $(2,15-17)$. As computed tomography examinations have yet to be performed in our university, it was impossible to confirm the results.

Another large study group were veterinary consultations related to the skeletal system, in particular the assessment of post-traumatic changes and metabolic bone disease (MBD). In the literature, the medical condition most often mentioned as the cause of consultation is food-related secondary hyperparathyroidism, while post-traumatic changes are mentioned less frequently $(16,17)$. In our study, the prevalence of the above problems was reverse to that described in the literature. On the other hand, referring veterinarians only write a preliminary diagnosis when post-traumatic changes, gout or MBD are suspected. In most cases of skeletal deformations, abnormal movements and, occasionally, other clinical signs, the initial diagnosis was not established. This is the reason behind the disproportion between the high percentage of animals with clinical signs related to the skeletal system and the small percentage of initial diagnoses of this problem. As a result, post-traumatic changes and metabolic bone disease were most common findings in further diagnostic imaging tests. Two of the animals showed radiological signs of bone osteolysis, which is suggestive of bone malignancy or osteitis. The radiological examination alone was not always sufficient to achieve a final diagnosis, and in those cases further examination was recommended.

A disadvantage of this study was the fact that it was based on a retrospective analysis of cases. In particular, the effectiveness of ultrasound methods in the study group cannot be compared over the nine year period covered by the data, because the initial examinations used 5-7.5-12 $\mathrm{MHz}$ probes, whereas in later years a $22 \mathrm{MHz}$ probe was used. Although X-ray and ultrasound examinations gave negative results in nearly $30 \%$ of cases, the results of similar tests performed today might give a completely different picture. On the other hand, as mentioned earlier, most of the problems reported by pet owners were general and non-specific, and imaging in such cases may not always be effective.

These difficulties were generally due to the small size of the animals, which made it very difficult to obtain satisfactory images. According to other authors, the identification of individual organs on an ultrasound scan can be problematic even in relatively large animals (1, $8)$. Only recent publications show a much higher effectiveness of ultrasound examination in small individuals (3). By analyzing individual publications from 1991-2015 concerning ultrasound evaluation of normal body organs in lizards, it can be concluded that the number of reports on the possibility of imaging specific coelomic organs is growing. This is explained by major technical advances in ultrasound equipment and increasingly detailed imaging capabilities. These conclusions are also valid for the retrospective analysis of the cases presented in this paper.

\section{References}

1. Banzato T., Hellebuyck T., Van Caelenerg A., Saunders J. H., Zotti A.: A review of diagnostic imaging of snakes and lizards. Vet. Rec. 2013 a, 173, 43-49.

2. Banzato T., Selelri P., Zotti A. L.: Comparative evaluation of the cadaveric and computed tomographic features of the coelomic cavity in the green iguana (Iguana iguana), black and white tegu (Tupinam bismerianae) and bearded dragon (Pogona vitticeps). Anat. Histol. Embryol. 2013 b, 42, 453-460.

3. Bucy D. S., Sanchez-Migallon Guzman D., Zwingenberger A. L.: Ultrasonographic anatomy of bearded dragons (Pogona vitticeps). J. Am. Vet. Med. Assoc. 2015, 246, 868-876.

4. Funk R. S.: Lizards reproductive medicine and surgery. Vet. Clin. N. Am. - Exotic 2002, 5, 579-613.

5. Gartell B. D., Girling J. E., Edwards A., Jones S. M.: Comparison of noninvasive methods for the evaluation of female reproductive condition in the large viviparous lizards, Tiliqua nigrolutea. Zoo Biol. 2002, 21, 253-268.

6. Gnudi G., Volta A., Ianni E. Di, Bonazzi M., Manfredi S., Bertoni G.: Use of ultrasonography and contrast radiography for snake gender determination. Vet. Radiol. Ultrasound 2009, 50, 309-311.

7. Grosset C., Daniaux L., Sanchez-Migallon Guzman D., Scott Weber III E., Zwingenberger A., Paul-Murphy J.: Radiographic anatomy and barium sulfate contrast transit time of the gastrointestinal tract of bearded dragons (Pogona vitticeps). Vet. Radiol. Ultrasound 2014, 55, 241-250.

8. Holland M. F., Hernandez-Divers S. J., Frank P. M.: Ultrasonographic appearance of the coelomic cavity in healthy green iguanas. J. Am. Vet. Med. Assoc. 2008, 233, 590-596.

9. Kemp S. D., Panciera D. L., Larson M. M., Saunders G. K., Werre S. R.: A comparison of hepatic sonographic features and histopathological diagnosis in canine liver disease: 138 cases. J. Vet. Intern. Med. 2013, 27, 806-813.

10. Love N., Douglass J. P., Lewbart G., Stoskopf M.: Radiographic and ultrasonographic evaluation of egg retention and peritonitis in two green iguanas (Iguana iguana). Vet. Radiol. Ultrasound 1996, 37, 68-73.

11. Łojszczyk-SzczepaniakA., Śmiech A., Chlebicka N., Szczepaniak K. O., KlimiukP.: First case of intestinal leiomyosarcoma in a bearded dragon - ultrasonographic findings. Med. Weter. 2016, 72, 273-336.

12. Mans C.: Clinical update on diagnosis and management of disorders of the digestive system of reptiles. J. Exot. Pet Medicine 2013, 22, 141-162.

13. Martorell J., Espada Y., Ruiz De Gopegui R.: Normal echoanatomy of the redeared slider terrapin (Trachemys scripta elegans). Vet. Rec. 2006, 155, 417-420.

14. Melidone R., Knoll J. S., Parry N.: Preovulatory stasis and dystocia in oviparous lizards. Vet. Med. 2008, 11, 595-598.

15. Murray M. J.: Pneumonia and lower respiratory tract diseases, [in:] Mader D (ed): Reptile Medicine and Surgery. Elsevier, St. Louis, Missouri 2006, p. 865-877 .

16. Schumacher J., Toal R. L.: Advanced radiography and ultrasonography in reptiles. Semin. Avian Exot. Pet Med. 2001, 10, 162-168.

17. Silverman S.: Diagnostic Imaging. [in:] Mader D. (ed): Reptile Medicine and Surgery. Elsevier, St. Louis, Missouri 2006, p. 471-489.

18. Sokal R. R., Roholf F. J.: Biometry, [in:] The Principles and Practices of Statistics in Biological Research. Freeman W. H., New York 1994.

19. Suedmyer K.: Noninfectious diseases of reptiles. Semin. Avian Exot. Pet Med. 1995, 4, 56-60.

20. Wang K. Y., Panciera D. L., Rukibat R. K. Al, Radi Z. A.: Accuracy of ultrasoundguided fine-needle aspiration of the liver and cytologic findings in dogs and cats: 97 cases (1990-2000). J. Am. Vet. Med. Assoc. 2004, 224, 75-78.

Corresponding author: Anna Lojszczyk-Szczepaniak, DVM, PhD, Pracownia Radiologii i Ultrasonografii, Katedra i Klinika Chirurgii Zwierzat, Głęboka 30, 20-950 Lublin, Poland; e-mail: anna.lojszczyk@gmail.com 